\title{
Structure of a spin-crossover Fe(II)-1,2,4-triazole polymer complex gel in toluene. Small angle neutron scattering and viscoelastic studies
}

Coro Echeverría ${ }^{a}$, Miguel Rubio ${ }^{a}$, Geoffrey R. Mitchell ${ }^{b}$ and Daniel López ${ }^{\mathrm{a}, \mathrm{b}, *}$

${ }^{a}$ Instituto de Ciencia y Tecnología de Polímeros, ICTP-CSIC, calle Juan de la Cierva, 3 Madrid 28006, Spain

${ }^{\mathrm{b}}$ Polymer Science Centre, University of Reading, Whiteknights, Reading, UK

*Corresponding author e-mail: daniel.1.g@csic.es 


\begin{abstract}
In this paper we report the study of metallo-organic polymer gels of [Fe(II) (4octadecyl-1,2,4-triazole $\left.)_{3}\left(\mathrm{ClO}_{4}\right)_{2}\right]_{\mathrm{n}}$ in toluene. A thorough investigation of the molecular structure and the viscoelastic properties of the metallo-organic polymer gels in toluene has been carried out by small angle neutron scattering and controlled stress oscillatory rheology as a function of temperature and concentration in order to understand the selfassembly process leading to the gel formation. The results obtained point out a side-byside aggregation of the individual metallo-organic polymer chains, behaving as "living" polymers, into fibres of different cross-sections as responsible for the gelation of these systems.
\end{abstract}

\title{
INTRODUCTION
}

Metallo-organic polymer chains of $\mathrm{Fe}(\mathrm{II})$ ions bridged by triazole ligands exhibit thermally induced transitions between two different electronic states: a diamagnetic $(S=0)$ low spin state and a paramagnetic $(S=2)$ high spin state. This spin transition is usually accompanied by changes in the optical and other physico-chemical properties[19]. This behaviour has attracted a great deal of interest due to the potential applications of these systems in the design of multi functional materials with interesting thermal, optical, magnetic and electronic properties[10-14].

The main problem that needs to be overcome for the development of new functional materials is how to preserve the bulk properties of the metallo-organic polymer in the solid state, in systems suitable for technological applications. For this purpose strategies such as Langmuir-Blodgett techniques[15, 16], layer-by-layer assembly[17], electron beam lithography[18] and self-organization of molecular spin-transition units into hierarchically organized domains across different length scales, have been applied[5, 19].

In this context, the ability to isolate and preserve such linear metallo-organic polymers in solution forming gels and polymer films constitutes a route to obtain novel functional materials, although this methodology has been little developed so far [20-24]. 
In previous works[6,7], we have reported on the structure of a metallo-organic polymer in the solid state using DSC and WAXD and SAXS techniques. We have also confirmed that the metallo-organic polymer based on chains of Fe(II) ions bridged by triazole ligands was able to produce thermoreversible gels in some organic solvents (i.e. toluene, cis- and trans-decalin). The obtained gels showed sol-gel transition temperatures that can be tuneable through the control of the polymer concentration and solvent type. However, the results obtained in these studies pointed out the structural differences between systems and different gelation mechanisms in the preparation of metallo-organic polymer in different solvents, which suggest the necessity to deepen in the structural characterization of these forming gels. Other studies[25, 26] have evidenced the fibril nature of analogous metallo-organic polymer gels through AFM and TEM studies, but the aggregation mechanisms conducting to the fibre formation remains to be understood. Consequently, the aim of this paper is the examination of the resulting molecular structure of the metallo-organic polymer gels in toluene, one of the solvents in which this polymer forms thermoreversible gels as a function of temperature and concentration by small angle neutron scattering (SANS). This allows us to understand the self-assembly process and the gelation mechanism of this polymer complex gel. In addition, the rheological behaviour of the system at different temperatures is also analysed in order to verify the mechanism proposed.

\section{EXPERIMENTAL PART}

\section{Materials}

For the synthesis of 4-octadecyl-1,2,4-triazole (ODT), monoformylhydrazine (Aldrich) triethylorthoformate (Fluka), octadecylamine (Fluka) and 1-pentanol (Aldrich) were used. 1-pentanol was dried over anhydrous calcium sulphate and stored under nitrogen atmosphere before use. For the synthesis of the iron complex, iron(II) perchlorate (Aldrich) and ethanol (Merck) were used without previous purification. The solvent used for gel preparation was toluene (Merck).

\section{Sample preparation}

The metallo-organic polymer $\left[\mathrm{Fe}(\mathrm{II})(\mathrm{ODT})_{3}\left(\mathrm{ClO}_{4}\right)_{2}\right]_{\mathrm{n}}$ was obtained by a method reported previously[7] gels were prepared by mixing appropriate amounts of metallo- 
organic polymer and toluene at $65{ }^{\circ} \mathrm{C}$ until complete dissolution. Then, the formed solutions were quenched below $25^{\circ} \mathrm{C}$, the gelation temperature. As a result, transparent gels with concentrations ranging from $3 \%$ to $25 \%(\mathrm{~g} / \mathrm{mL})$ were obtained.

\section{Small Angle Neutron Scattering (SANS)}

SANS experiments were carried out using LOQ small angle diffractometer at the ISIS Pulse Neutron Source (STFC facility Rutherford Appleton Laboratory, Didcot, UK) with a $12 \mathrm{~mm}$ diameter incident beam[27]. The neutron wavelength, $\lambda$, was employed in a range of $2.2 \AA-10 \AA$, giving a scattering vector range $|q|$ of $0.009 \AA^{-1}$ $0.25 \AA^{-1}$ where $q=4 \pi \sin \theta / \lambda$ and $2 \theta$. Scattering profiles of each sample were accumulated for 60 minutes in order to obtain a good signal to noise ratio. The data were collected on the two-dimensional ORDELA area detector. The obtained raw data were corrected for the absorption and scattering from the sample container and converted to an absolute scale using established procedures[28-30]. The twodimensional data were reduced to one-dimensional differential scattering cross-section $((\mathrm{d} \Sigma / \mathrm{d} q)(q))$, as a function of $|q|$. Data analysis was performed in a non-linear least squares data-fitting program (FISH)[31]. The evaluation of incoherent scattering was incorporated into the data fitting method.

\section{Viscoelastic measurements}

Rheological studies were performed using a controlled stress oscillatory rheometer TA Instruments ARG2. $40 \mathrm{~mm}$ diameter parallel plates were used for all the samples. Samples were loaded onto the stage (peltier plate) and the upper plate was lowered slowly until the desired sample position was reached. To minimise solvent evaporation, a solvent trap device was used. The linear viscoelastic region (LVR) was located with the aid of a torque sweep test at a constant non-destructive frequency of 1 $\mathrm{Hz}$. Then, frequency sweep tests from $40 \mathrm{~Hz}$ to $0.01 \mathrm{~Hz}$ in the LVR at temperatures from $55{ }^{\circ} \mathrm{C}$ to $80{ }^{\circ} \mathrm{C}$ were carried out.

\section{RESULTS AND DISCUSSION}

In order to investigate the molecular structure of the metallo-organic polymer gels in toluene, SANS experiments were performed at different concentrations and temperatures. Figure 1 shows the small angle neutron scattered intensity as a function of $q$ plotted in a $\log -\log$ scale for a sample of concentration $3 \%(\mathrm{~g} / \mathrm{mL})$ at temperatures in 
the range from $0{ }^{\circ} \mathrm{C}$ to $100^{\circ} \mathrm{C}$. As can be seen from Fig 1(A), the intensity scattered at high angles is qualitatively similar, indicating that structural features at short distances do not vary with temperature. However, at low angle values, the scattering intensity is dependent on the temperature. Actually, the scattered intensity increases as the temperature decreases probably due to an aggregation process of the metallo-organic polymer chains. A significant change in the scattered intensity profile between 20 and $25^{\circ} \mathrm{C}$ is also evident that might be related to a possible structural transition.

For the sake of clarity, the scattering curve of the sample at a concentration of $3 \%$ $(\mathrm{g} / \mathrm{mL})$ at $25^{\circ} \mathrm{C}$ is depicted in Figure 1B. In the plot, three different scattering regimes can be observed: i) a first regime at $0.01<q<0.05$ values where the scattered intensity varies as a function of $q^{-1}$, which corresponds fairly well to randomly oriented solidrods, ii) a second regime at intermediate $q$ values $(0.05<\boldsymbol{q}<0.1)$ where the scattered intensity shows a $\mathrm{I}(q) \approx q^{-2}$ dependence, and iii) a third regime at high $q$ values $(q>0.1)$ where the scattered intensity presents a power law dependence of $\mathrm{I}(q) \approx q^{-4}$, and that corresponds to the Porod region containing important information about the local rod cross-section[32, 33].
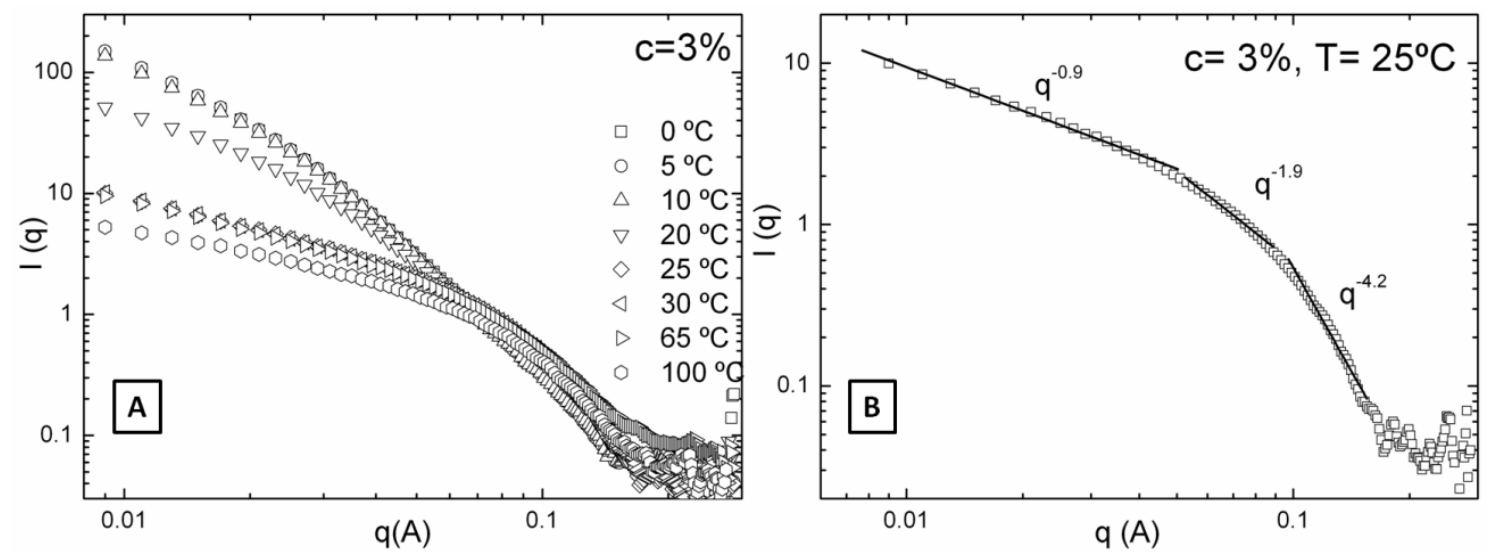

Figure 1: (A) Intensity scattered by a metallo-organic polymer gel in toluene at a concentration of $3 \%(\mathrm{~g} / \mathrm{mL})$ at different temperatures. (B) Intensity scattered by sample at $3 \%$ of concentration and $25^{\circ} \mathrm{C}$.

The scattering curves at different concentrations and temperatures are represented as kratky plots $\left(q^{2} \mathrm{I}\right.$ vs $q$ ) in Figure 2. This type of representation produces "bell-shaped" scattering curves with a linear dependency at low $q$ values, which is characteristic of the scattering behaviour of rod-like structures[34, 35]. The average cross-section and the 
length of the solid rods responsible for the scattering curves can be determined from the linear part in the kratky representations.

Figure 2(A) displays kratky curves for the gels at concentrations of 1.5, 3, 6 and 12\% $(\mathrm{g} / \mathrm{mL})$. This allows us to evaluate the effect of concentration on the molecular structure of the gel. "Bell-shaped" curves are observed for all the concentrations, with the maximum of the peaks at approximately the same $q$ value. Hence, despite the fact that the increase of concentration gives rise to more intense peaks, the rod-like cylindrical structure of the metallo-organic polymer chains in the gels appears to be independent of the concentration of the samples. However and as it is shown in Figure 2(B), where the scattering curves of the formed gels are represented as a function of temperature, there is an effect of the temperature on the molecular structure of the gels. Although the "bell-shaped" curves are observed for each temperature, which indicates that the rodlike structure is also independent of temperature, there is a shift of the peak toward lower $q$ values at temperatures below $25^{\circ} \mathrm{C}$, suggesting a different supra molecular organization of the metallo-organic polymer chains, as we will discuss in the following sections.
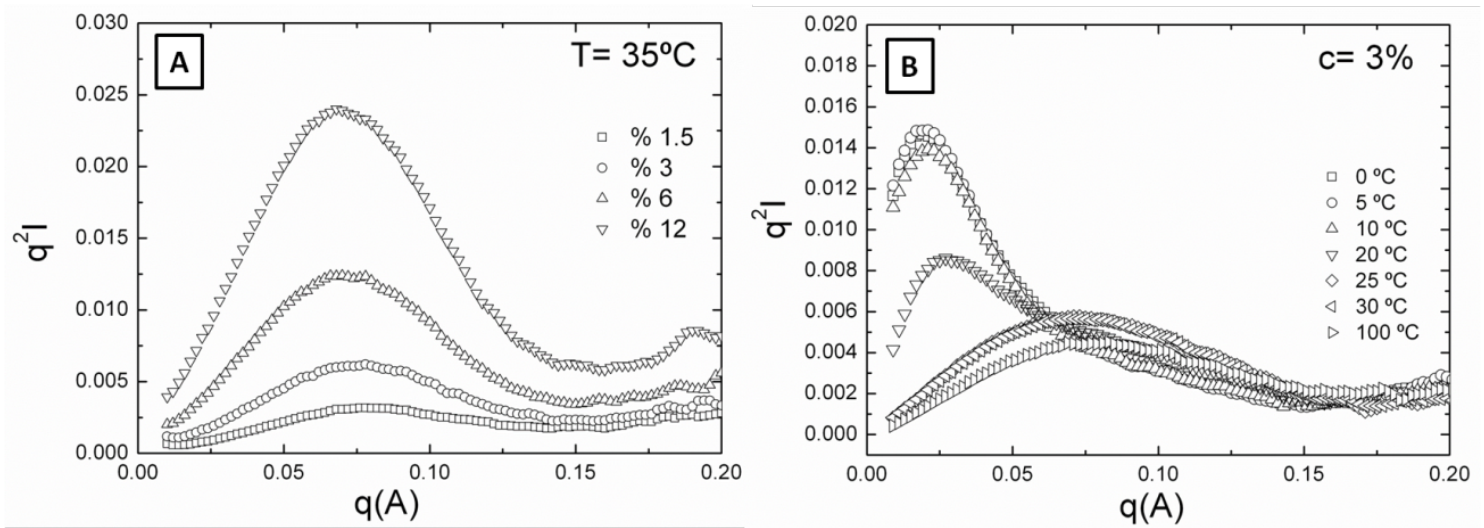

Figure 2: $q^{2}$ I vs $q$ Neutron scattering curves obtained for the metallo-organic polymer in toluene at (A) $35^{\circ} \mathrm{C}$ and concentrations of $1.5,3,6$ and $12 \%(\mathrm{~g} / \mathrm{mL})$ and $(B)$ for of the gel with $3 \%(\mathrm{~g} / \mathrm{mL})$ concentration at different temperatures.

\section{Molecular structure at $\mathrm{T}>25^{\circ} \mathrm{C}$}

Figure 3 shows the experimental scattering curve of the metallo-organic polymer/toluene system with a concentration of $3 \%(\mathrm{~g} / \mathrm{mL})$ measured at $30^{\circ} \mathrm{C}$. The experimental curve can be fitted by considering a solid-cylinder model of cross-section 
radius $r_{c}$ and length of the cylinder $L$. For $q L>1$ and $L>r_{c}$ the theoretical scattering intensity of a solid cylinder is written as[36, 37]:

$q^{2} I(q)=C_{p} \mu_{L} \frac{4 J_{1}^{2}\left(q r_{c}\right)}{q^{2} r_{c}^{2}}\left[\pi q-\frac{2}{L}\right]$

where $\mu_{L}$ is the mass per unit length, $C_{p}$ solid cylinder concentration, $J_{l}$ is the first order Bessel function. As it can be observed in the plot, the theoretical equation fits reasonably well the experimental data in the $q$-region under study. The best fit was obtained for:

$C_{p} \mu_{L}=0.04 \pm 0.005 \mathrm{~g} \mathrm{~mol}^{-1} \AA^{-1}, r_{c}=18.2 \pm 0.5 \AA$ and $L=205 \pm 12 \AA$

The values obtained for the radius of the solid-cylinder are in good agreement with previous results reported in our group obtained by means of SAXS measurements for the solid metallo-organic polymer[6]. Those experiments allowed us to describe the system as layers of metal transition (II) ions intercalated between two layers of 4octadecyl-1,2,4-triazole with an inter-sheet spacing of $32 \AA$ (approximately double the cross-section radius of the solid cylinders determined in the current experiments). The same fitting procedure was followed for the system at different concentrations and in the range between 25 and $85{ }^{\circ} \mathrm{C}$. Table 1 collects the cross-section radius and length values determined for each gel.

The values obtained for the cross-section radius of the solid cylinders are in the range of 17-20 $\AA$ and can be explained by metallo-organic polymer fibres composed of one individual polymer chain.

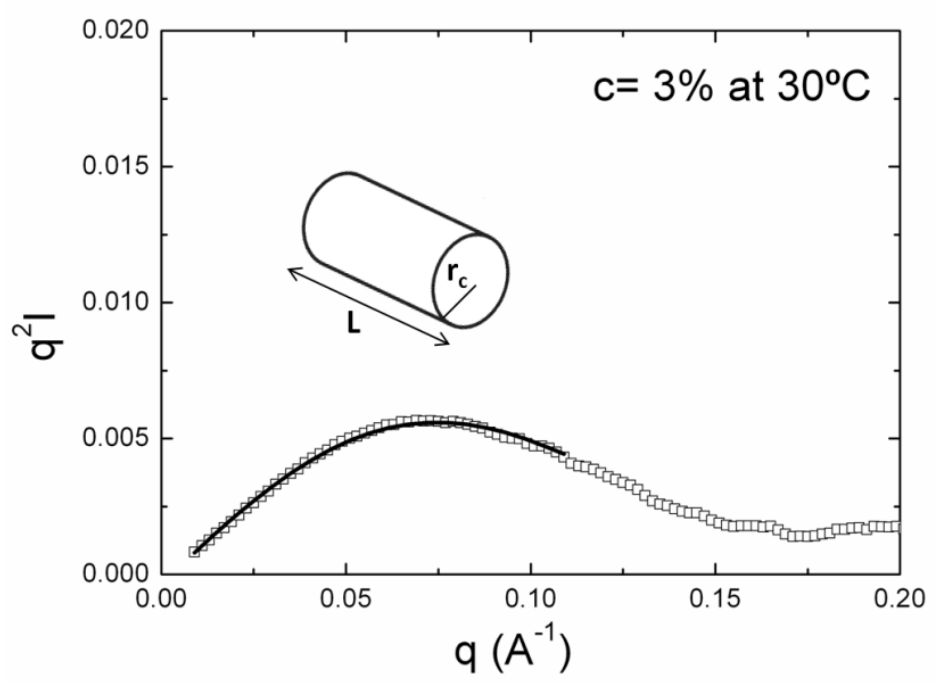


Figure 3: Kratky representation of the intensity scattered by a metallo-organic polymer gel in toluene at a concentration of $3 \%$ and $30^{\circ} \mathrm{C}$. The solid line represents the best fit obtained using equation 1 .

Table 1: $r_{c}, L$ and $C_{p} \mu_{L}$ obtained for the gels at concentrations of $3,6,12$ and $25 \%$ $(\mathrm{g} / \mathrm{mL})$ by fitting the experimental scattering curves to the solid-cylinder model of equation 1 .

\begin{tabular}{|c|c|c|c|c|}
\hline & & \multicolumn{3}{|c|}{ Kratky representation } \\
\hline $\mathrm{C}(\mathrm{g} / \mathrm{ml})$ & $\mathrm{T}\left({ }^{\circ} \mathrm{C}\right)$ & $L(\AA)$ & $r_{c}(\AA)$ & $C_{p} \mu_{L}$ \\
\hline \multirow{5}{*}{3} & 25 & $217 \pm 15$ & 18.5 & 0.04 \\
& 30 & $205 \pm 12$ & 18.7 & 0.04 \\
& 65 & $158 \pm 8$ & 17.8 & 0.04 \\
& 100 & $87 \pm 3$ & 17.8 & 0.03 \\
\hline \multirow{6}{*}{6} & 55 & $255 \pm 32$ & 20.0 & 0.10 \\
& 65 & $156 \pm 16$ & 19.7 & 0.09 \\
& 75 & $73 \pm 3$ & 19.7 & 0.09 \\
& 85 & $65 \pm 1$ & 19.7 & 0.09 \\
\hline \multirow{6}{*}{12} & 25 & $118 \pm 41$ & 18.7 & 0.16 \\
& 35 & $141 \pm 16$ & 20.0 & 0.18 \\
& 45 & $118 \pm 13$ & 20.5 & 0.20 \\
& 55 & $63 \pm 4$ & 20.0 & 0.20 \\
& 65 & $45 \pm 2$ & 19.7 & 0.18 \\
& 75 & $42 \pm 2$ & 18.7 & 0.16 \\
& 85 & $40 \pm 1$ & 18.7 & 0.17 \\
\hline \multirow{6}{*}{25} & 25 & $45 \pm 4$ & 18.7 & 0.28 \\
& 35 & $34 \pm 1$ & 20.2 & 0.42 \\
& 45 & $28 \pm 1$ & 20.2 & 0.43 \\
& 55 & $24 \pm 1$ & 18.9 & 0.38 \\
& 65 & $23 \pm 1$ & 18.7 & 0.34 \\
& 75 & $21 \pm 1$ & 18.1 & 0.32 \\
& 85 & $19 \pm 1$ & 18.2 & 0.34 \\
\hline
\end{tabular}

Effect of temperature on discrete metallo-organic polymer fibres above $25{ }^{\circ} \mathrm{C}$ : Application of Cate's theory

Individual fibres of the metallo-organic polymer in the gel state undergo a decrease in length as the temperature increases for all the concentrations (see Table 1). This behaviour is probably due to a local rupture of the polymer chains as a consequence of the change in the coordination of the $\mathrm{Fe}$ (II) ions as reported before for analogous $\mathrm{Co}(\mathrm{II})$ triazole polymer complexes[38]. As we will present further in the manuscript, the 
rheological behaviour of the system points out the Maxwell type relaxation processes. Additionally, as it has been stated before, neutron scattering experiments suggest that the molecular structure of the metallo-organic polymer in the gel state is fibre-like. Considering these two results, it should be possible to describe the metallo-organic complex as a "living" polymer, where only a characteristic relaxation time is involved. In that case, the system could be studied through Cate's theory[39, 40] in which the characteristic relaxation time is a contribution of two characteristic relaxations processes associated to the reptation (rep) of the polymer chains and to the scission (break) of the fibres, $\tau=\left(\tau_{\text {rep }} \cdot \tau_{\text {break }}\right)^{1 / 2}[41,42]$.

Cate's theory[39, 40] considers that the relaxation mechanism of the metallo-organic complex is a scission/recombination mechanism. Due to this mechanism the length distribution of the chains (fibres) depends on the temperature, the concentration as well as on the scission energy of the chains. Among others, the main hypothesis of this theory is that the scission energy is independent of the concentration. Therefore, it is possible to calculate the distribution in length of fibres that obeys Boltzman statistics in the thermodynamic equilibrium, through living polymers:

$<L>\sim \varphi^{\alpha} \exp \frac{E_{s c i}}{2 R T}$

Being $\varphi$ the fibre volume fraction, $\alpha$ an exponent which depends on the long-range statistics of the filaments and $R=8.31447 \mathrm{~J} / \mathrm{mol} \cdot \mathrm{K}$

Figure 4 represents the evolution of the average length with temperature for metalloorganic polymer gels at concentrations of 6,12 and $25 \%(\mathrm{~g} / \mathrm{mL})$ as determined from SANS measurements. As can be observed, the experimental points follow two different linear dependencies that crossover at 75,71 and $45{ }^{\circ} \mathrm{C}$ for concentrations of 6,12 and $25 \%(\mathrm{~g} / \mathrm{mL})$, respectively. This crossover temperature corresponds to the separation between two different relaxation behaviours: At high temperatures the scission energy obtained $E_{s c i} \approx 36-54 \mathrm{KJ} / \mathrm{mol}$ is almost independent of the gel concentration, suggesting that we are dealing with rather "living-like polymers". Therefore, the scission energy will correspond to the breakage or scission process of individual fibres (as seen in Figure 4). Nevertheless, at low temperatures the scission energy decreases as the concentration increases, which means that as the concentration increases a higher increment of temperature is necessary to provoke the same length variation. This result 
can be explained considering that the relaxation of the polymer at these temperatures implies a reptation process and further scission of the fibres.

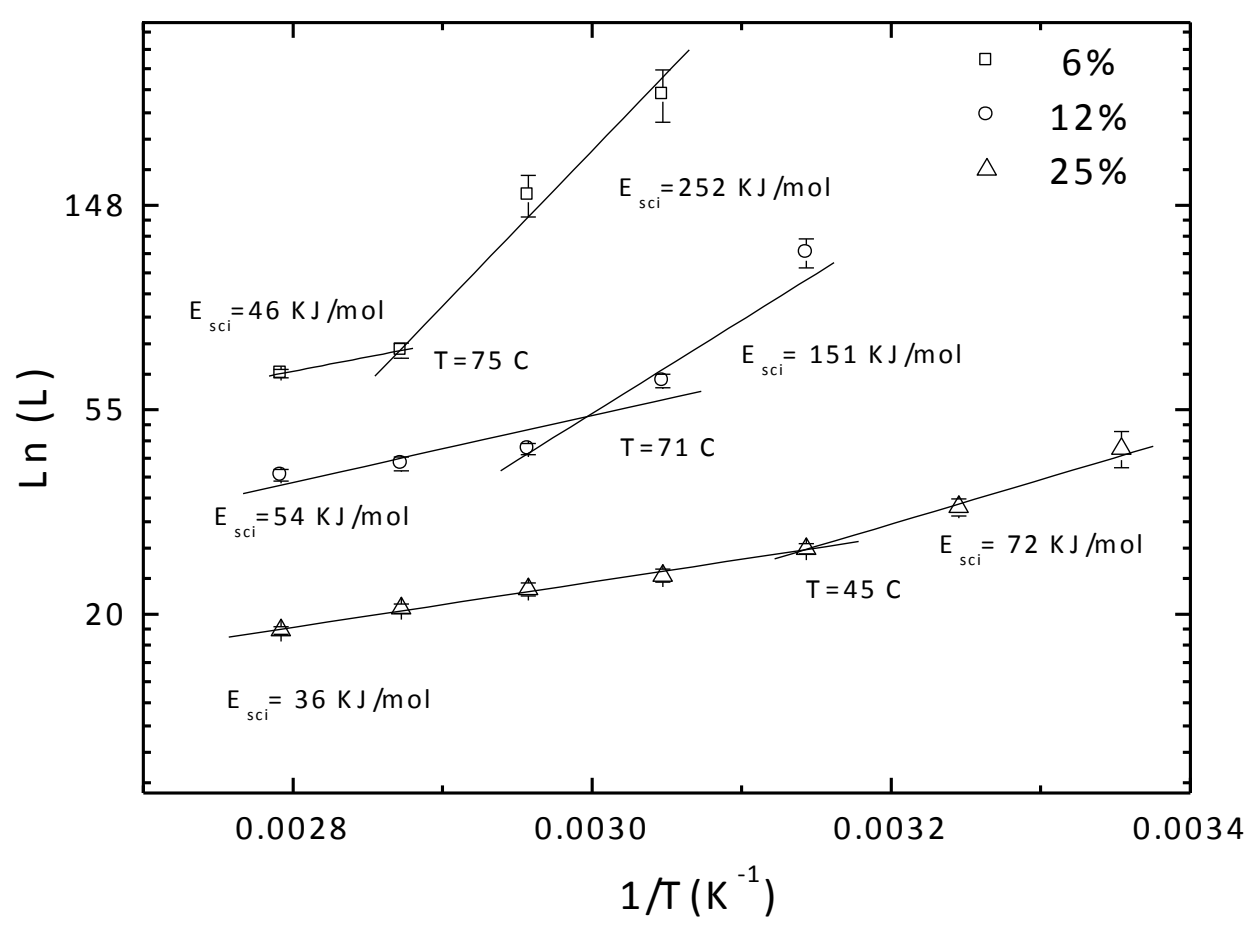

Figure 4: Evolution of the length distribution with temperature of the metallo-organic fibres for samples at concentrations of 6,12 and $25 \% \mathrm{~g} / \mathrm{ml}$ in toluene.

Molecular structure at $T<25^{\circ} \mathrm{C}$

In Figure 5 the Kratky plots for gels of concentration $3 \%(\mathrm{~g} / \mathrm{mL})$ measured at $0{ }^{\circ} \mathrm{C}$ are depicted. As mentioned before, the peaks of the scattering curves appear at lower values of $q$ suggesting the presence of fibrils or cylinders of larger diameters, probable formed due to the aggregation of single metallo-organic polymer chain fibres. Therefore, it is not possible to fit our experimental data using eq (1) considering single polymer chain fibres as shown in Fig. 3.

In a first attempt to fit the experimental data we tried a model that considers a twopopulation system of fibres, one of the population formed by single chain molecules and the other one formed by the aggregation of different chains, with radii $r_{c}$ and $R$ and linear masses $\mu_{\mathrm{L} 1}$ and $\mu_{\mathrm{L} 2}$, respectively given by (for $\mathrm{L}>\mathrm{r}$ ): 
$q^{2} I(q)=x\left\{C_{p} \mu_{L 1} \pi \frac{4 J_{1}^{2}\left(q r_{c}\right)}{q^{2} r_{c}^{2}}\right\}+(1-x)\left\{C_{p} \mu_{L 2} \pi \frac{4 J_{1}^{2}(q R)}{q^{2} R^{2}}\right\}$

where $\mathrm{x}$ is the fraction of the population of single chain fibres.

The best fit of the experimental data of the gel sample with a concentration of $3 \%$ $(\mathrm{g} / \mathrm{mL})$ at $0{ }^{\circ} \mathrm{C}$ with equation (3) (see Figure 5A) gives: $r_{c}=18 \AA, R=59 \AA$ and $x=0.65$ $C_{p} \mu_{L 1}=0.04 C_{p} \mu_{L 2}=0.087$. The value obtained for $r_{c}$ is very close to the fibrils radius previously found for the same sample at $30{ }^{\circ} \mathrm{C}$ suggesting the presence of the same type of elementary fibrils. On the other hand, the value obtained for $C_{p} \mu_{L 2}$ is twice the one obtained for $\mathrm{C}_{\mathrm{p}} \mu_{\mathrm{L} 1}$ suggesting the association of two elementary chains to form a thicker fibre with twice the linear mass. However, the value obtained for $R$ is three times the value obtained for $r_{c}$, indicating that the systems could also be the association of three elementary fibrils.

To verify whether these two possible molecular structures could fit the experimental data, the theoretical scattering curves were more explicitly written by considering Oster and Riley form factor for a collection of $n$ cylinders[43]:

$I(q)=\frac{\pi \mu_{L}}{q n^{2}} \varphi\left(q r_{c}\right) \sum_{j} \sum_{k} J_{0}\left(q r_{j k}\right)$

where $n$ in the number of rod-like entities and $\varphi(q r)$ their cross-section scattering.

For an aggregate formed with two cylinders $n=2$ (see Figure 5B), the equation (4) becomes:

$q^{2} I(q)=C_{p} \mu_{L} \pi \frac{4 J_{1}^{2}(q R)}{q^{2} R^{2}}\left(2+2 J_{0}(q R)\right)$

and considering a two population system of individual solid cylinder structures and this kind of aggregates with $n=2$, the scattering intensity gives:

$q^{2} I(q)=x\left\{C_{p} \mu_{L 1} \pi \frac{4 J_{1}^{2}\left(q r_{c}\right)}{q^{2} r_{c}^{2}}\right\}+(1-x)\left\{C_{p} \mu_{L 2} \pi \frac{4 J_{1}^{2}(q R)}{q^{2} R^{2}}\left(2+2 J_{0}(q R)\right)\right\}$

where $R=2 r_{c}+1$, being 1 the distance between cylinders (see the model in Fig. 5B).

By fixing $C_{p} \mu_{L 1}=0.04$, the best fit obtained for this second molecular model gives the following values for the model parameters: $r_{c}=19 \AA, R=55 \AA$ and $x=0.07 C_{p} \mu_{L 1}=$ $0.04, C_{p} \mu_{L 2}=0.176$. 
The values obtained for $r_{c}$ and $R$ are consistent with the model proposed. However, the mass per unit length $C_{p} \mu_{L 2}$ is 4 times greater than $C_{p} \mu_{L 1}$, showing an incoherence with the proposed model of aggregates formed with two single chains. Furthermore, it is possible to deduced from the value of $R=2 r_{c}+1$ that this molecular model present an inter-fibril distance of $1=16.52 \AA$. This distance is similar to the fibril $r_{c}$ which lead us to consider the possibility of the presence of a third cylinder in the agglomerated structure. Therefore, we proposed a third model that considers the scattering of a mixture of two population of rods with radii $r_{c}$ and a population formed by the parallel association (see Figure 5C) of three elementary fibrils, $n=3$. Therefore, considering the Oster and Riley form factor[43] for a system with $n=3$ parallel fibrils, equation (4) becomes:

$q^{2} I(q)=C_{p} \mu_{L} \pi \frac{J_{1}^{2}(q R)}{q^{2} R^{2}} q\left(3+4 J_{0}\left(q\left(2 r_{c}+l\right)\right)+J_{0}\left(q\left(4 r_{c}+l\right)\right)\right.$

where $l$ is the distance between the cylinders; and considering the two population system as before:

$q^{2} I(q)=x\left\{C_{p} \mu_{L 1} \pi \frac{4 J_{1}^{2}\left(q r_{c}\right)}{q^{2} r_{c}^{2}}\right\}+(1-x)\left\{C_{p} \mu_{L 2} \pi \frac{J_{1}^{2}(q R)}{q^{2} R^{2}} q\left(3+4 J_{0}\left(q\left(2 r_{c}+l\right)\right)+J_{0}\left(q\left(4 r_{c}+l\right)\right)\right\}\right.$

Fixing the masses per unit length, $C_{p} \mu_{L 1}=0.04, C_{p} \mu_{L 2}=0.12$, the best fit obtained by applying equation (8) gives the following values for the model parameters: $r_{c}=17.04 \AA$ and $l=3.30 \AA$. The value found for $r_{c}$ is in the range of that obtained for individual solid-cylinders, however, the fitting curve does not fit properly the experimental data. Thus, we considered another organization of the molecular structure by proposing a last molecular model formed by individual solid cylinders and a second population formed by aggregates of three elementary fibrils but disposed in a triangular arrangement. Considering the Oster and Riley form factor for a system with $n=3$ fibrils assembled as a triangular geometry and the presence of the two population system indicated, equation (4) becomes:

$$
q^{2} I(q)=x\left\{C_{p} \mu_{L 1} \pi \frac{4 J_{1}^{2}\left(q r_{c}\right)}{q^{2} r_{c}^{2}}\right\}+(1-x)\left\{1.3 * C_{p} \mu_{L 2} \pi \frac{J_{1}^{2}(q R)}{q^{2} R^{2}} q\left(1+2 J_{0}(q R)\right)\right\}
$$

where $R=2 r_{c}+1$. 
The theoretical curve obtained through eq (9) fits fairly well the experimental data (see Figure 5D). Fixing the masses per unit length at $C_{p} \mu_{L 1}=0.04$ and $C_{p} \mu_{L 2}=0.12$, the best fit obtained gives the following results: $r_{c}=24.06 \AA, R=57,23 \AA, 1=9.11 \AA, x=0.22$

The results obtained clearly show that the metallo-organic polymer chains that adopt rod-like conformations in solutions of toluene, aggregate at low temperatures to produce solid-like gels irrespective of the number of chains and their spatial disposition within the aggregates. The best fit for the metallo-organic polymer gel in toluene at a concentration of $3 \%(\mathrm{~g} / \mathrm{mL})$ and temperature of $0{ }^{\circ} \mathrm{C}$ suggests that the composition of the systems is $22 \%$ of individual fibrils and $78 \%$ aggregates. Results obtained for samples at a concentration of $3 \%$ measured at temperatures of 0,5 and $10{ }^{\circ} \mathrm{C}$ are summarized in Table 2.
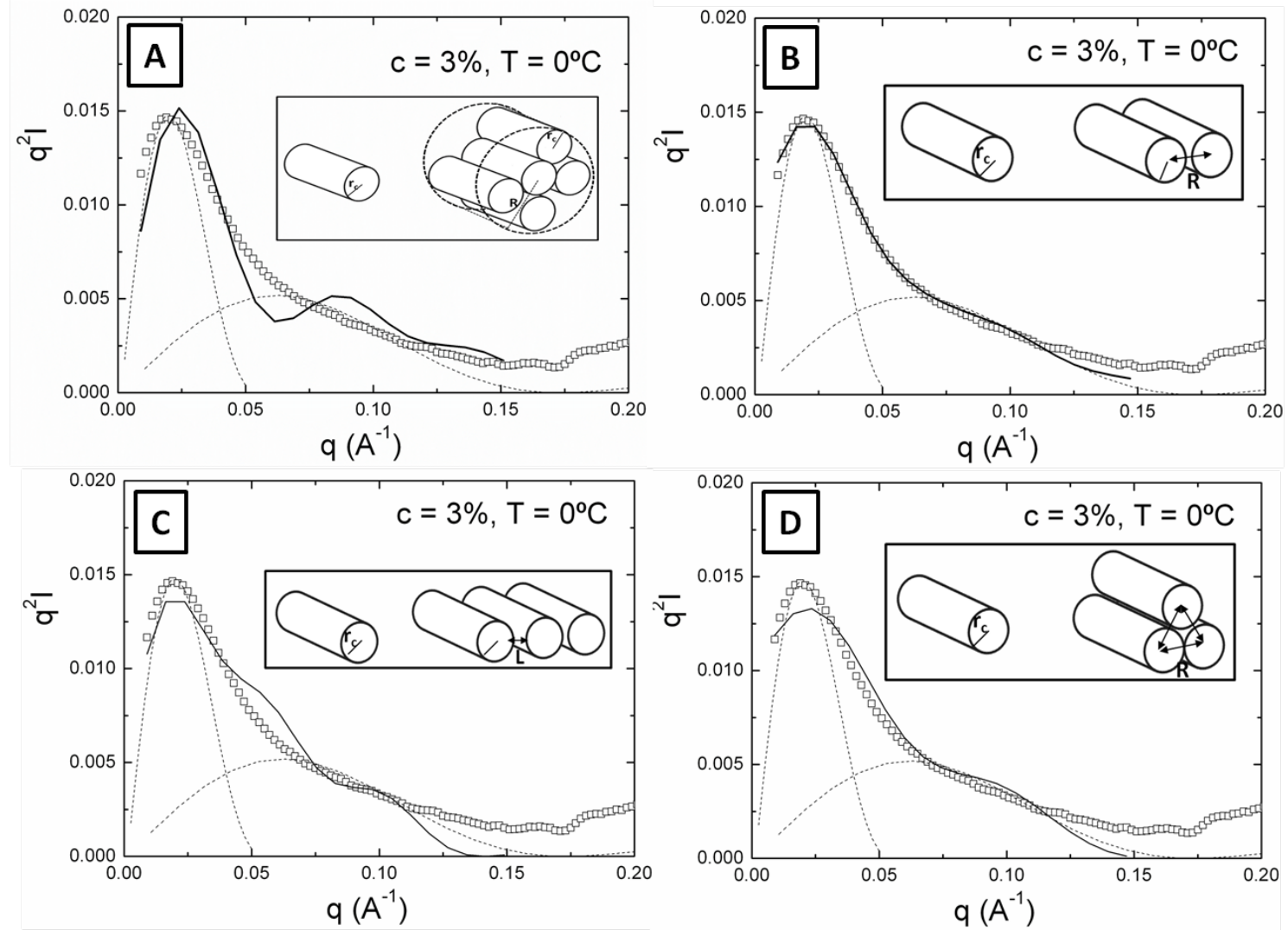

Figure 5: Neutron scattering curves obtained for the polymer at $3 \%$ of concentration and at $0{ }^{\circ} \mathrm{C}$ by means of Kratky representations. The solid line represents the best fit obtained using (A) equation 3, (B) equation 6, (C) equation 8 and (D) equation 9. Dash lines, that are guides for the eyes, correspond to each fibre population. 
Table 2 : Results obtained by fitting experimental scattering curves to eq. 9 for sample at $3 \%$ of concentration measured at $\mathrm{T}<25^{\circ} \mathrm{C}$.

\begin{tabular}{|c|c|c|c|}
\hline & \multicolumn{3}{|c|}{$\begin{array}{c}\text { Fitting to model (iv) } \\
\text { (Fig 5) }\end{array}$} \\
\hline $\mathrm{C}=3 \%$ & \multicolumn{3}{|c|}{ Temperature } \\
\hline Parameters & $\mathbf{0}^{\mathbf{}} \mathbf{C}$ & $\mathbf{5}^{\mathbf{}} \mathbf{C}$ & $\mathbf{1 0}^{\mathbf{}} \mathbf{C}$ \\
\hline$C_{p} \mu_{L 1}$ & 0.04 & 0.04 & 0.04 \\
\hline$C_{p} \mu_{L 2}$ & 0.12 & 0.12 & 0.12 \\
\hline$r_{c}(\AA)$ & 24.06 & 24.89 & 23.85 \\
\hline$R(\AA)$ & 57.23 & 58.14 & 55.96 \\
\hline$l(\AA)$ & 9.11 & 8.36 & 8.26 \\
\hline$x$ & 0.22 & 0.24 & 0.22 \\
\hline
\end{tabular}

\section{Viscoelastic properties of metallo-organic polymer gels in toluene.}

The gel state in the metallo-organic polymers/toluene system and its viscoelastic properties were investigated using oscillatory rheology. As it is known, a physical gel should be characterized rheologically by an elastic modulus at zero frequency[44]. Therefore, the metallo-organic polymer/toluene system was studied through frequency experiments represented in Figure 6. The measurements were carried out in the linear viscoelastic regime, as determined through oscillatory strain scans. In Figure 6, the evolution of storage modulus $\left(\mathrm{G}^{\prime}\right)$ and loss modulus $\left(\mathrm{G}^{\prime \prime}\right)$ with the applied frequency for samples of concentration $6 \%(\mathrm{~g} / \mathrm{mL})$ for temperatures between 55 and $80{ }^{\circ} \mathrm{C}$ are represented.

As it can be seen in the plot at 55 and $60{ }^{\circ} \mathrm{C}, \mathrm{G}^{\prime}$ and $\mathrm{G}^{\prime \prime}$ are found to be weakly dependent on frequency over the $0.1-0.01 \mathrm{~Hz}$ range, which indicates that the dominant viscoelastic relaxation modes of the network are found at lower frequencies. This means that the relaxation times of the networks, $\tau$, are longer. This also implies that the network junctions are relatively permanent. Such rheological behaviour is indicative of entangled polymeric melts and gels. 
At temperatures above $60{ }^{\circ} \mathrm{C}$ it is observed that $\mathrm{G}$ ' and $\mathrm{G}$ ' show the frequency dependency of liquid-like systems with a crossover point $\left(G^{\prime}=G^{\prime \prime}\right)$ at intermediate frequencies and a dependence of $G^{\prime} \propto \omega^{2}$ and $G^{\prime \prime} \propto \omega$ below the crossover point. The frequency at the crossover point $\mathrm{G}^{\prime}=\mathrm{G}^{\prime \prime}$ corresponds to the characteristic relaxation time of the polymer chains in the solvent. It is observed that as the temperature increases the crossover point increases toward lower values of frequency.

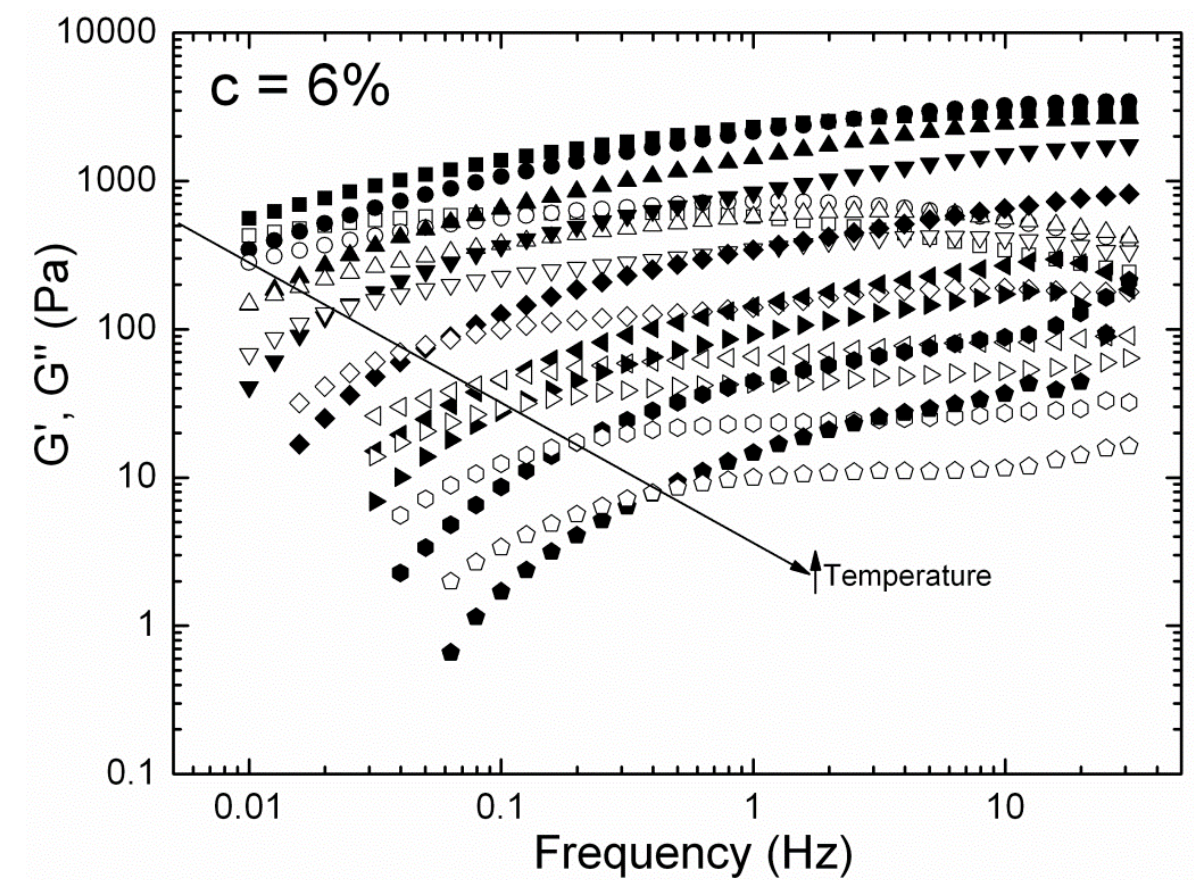

Figure 6: Evolution of G' (full symbols) and G' (empty symbols) with frequency for the $6 \%$ concentration sample at different temperatures: $\boldsymbol{\square} 55^{\circ} \mathrm{C}, \bullet 60^{\circ} \mathrm{C}, \boldsymbol{\Delta} 65^{\circ} \mathrm{C}, \boldsymbol{\nabla} 67.5^{\circ} \mathrm{C}$, $\bullet 70{ }^{\circ} \mathrm{C}, \varangle 72.5^{\circ} \mathrm{C}, 75^{\circ} \mathrm{C},-77.5^{\circ} \mathrm{C}$ and $\bullet 80^{\circ} \mathrm{C}$.

In figure 7 the Arrhenius representation of the relaxation time as a function of temperature is shown. It is clear that there are two different trends with the transition at around $71{ }^{\circ} \mathrm{C}$. This temperature indicates the separation between two different relaxation behaviours. At temperatures below $71^{\circ} \mathrm{C}$ the relaxation of the polymer chains presents activation energy, $E_{a}$, of $309.1 \mathrm{KJ} / \mathrm{mol}$ while at temperatures above $71^{\circ} \mathrm{C}$, the value is $177.94 \mathrm{KJ} / \mathrm{mol}$. These results are consistent with the results obtained by SANS experiments and commented previously. In both cases a transition in the relaxation of polymer chains is observed close to $70^{\circ} \mathrm{C}$. This behaviour could be related to changes in 
the interaction between metallo-organic polymer chains and their aggregationdisaggregation processes through the mechanisms shown in Figure 4.

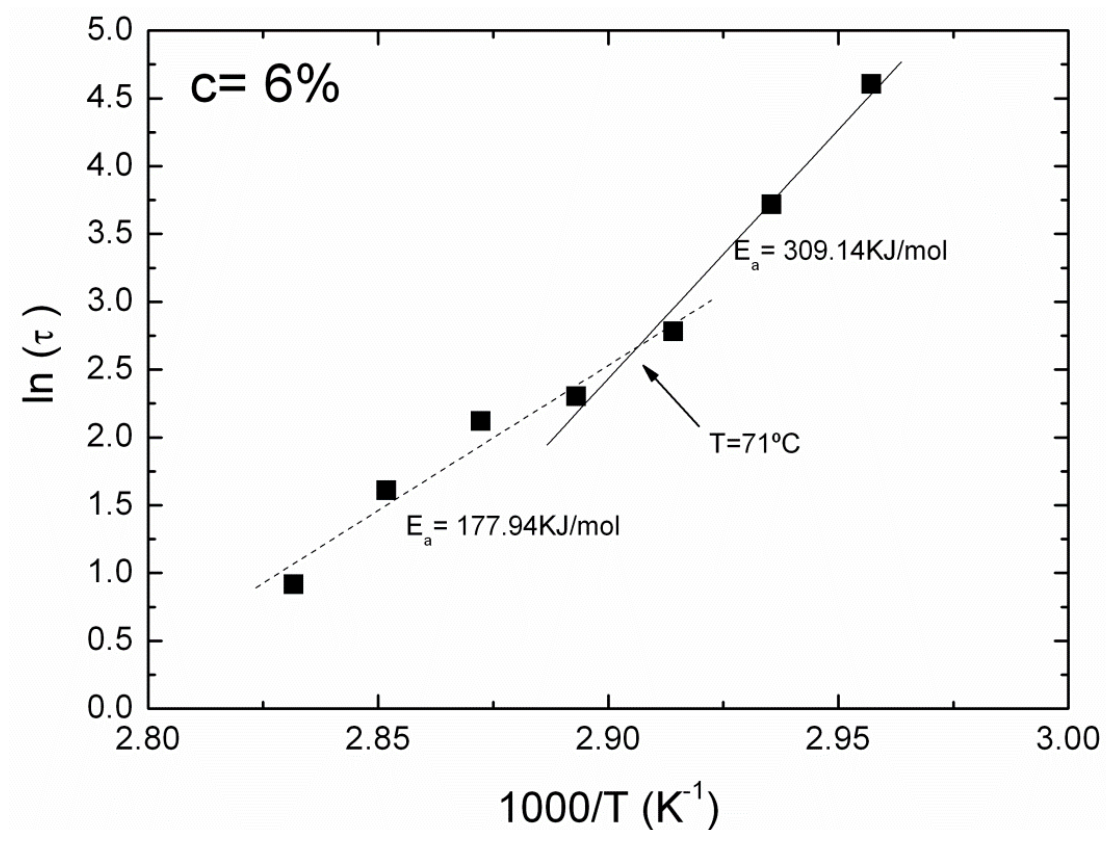

Figure 7: Arrhenius representation of the relaxation time as a function of reciprocal temperature for metallo-organic polymer gel in toluene with $6 \%$ of concentration.

\section{CONCLUSIONS}

The molecular structure of the metallo-organic polymer gel in toluene has been determined by SANS measurements. The structure is rather independent of concentration above the gelation threshold and consists basically of rod-like structures randomly oriented at temperatures above $25{ }^{\circ} \mathrm{C}$. The cross-section $(17-20 \AA)$ and the

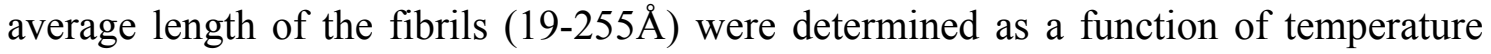
and concentration of the gels. Their values are consistent with isolated polymer chains of the metallo-organic polymer complex with varying lengths, obeying two different relaxations behaviours depending on temperature. At high temperatures the metalloorganic polymer chains behave as "living" polymers with scission energies of around 36-54 kJ/mol, corresponding to the breakage of individual chains. Nevertheless, at low temperatures, the relaxation of the polymer chains implies a reptation process and further scission of the chains, suggesting a side-by-side aggregation of the single metallo-organic polymer chains responsible for the gel formation. SANS results 
performed at the lowest temperatures suggest a molecular structure for the gels consisting of a two-population system of isolated polymer chains and fibres produced by a side-by-side aggregation of different number of individual chains. Viscoelastic experiments obtained from the same systems confirm these results.

\section{AKNOWLEDGMENTS}

Financial support from CICYT (MAT2008-01073 and MAT2011-24797) is gratefully acknowledged. D.L. thanks the Ministerio de Educación y Cultura (Spain) for his Salvador de Madariaga grant. The SANS experiments were possible through an award of beam time at the SFTF ISIS Facility. We thank Dr. Steve King for his help with the SANS experiments.

\section{BIBLIOGRAPHIC REFERENCES}

[1] Bousseksou A, Boukheddaden K, Goiran M, Consejo C, Boillot ML, Tuchagues JP. Dynamic response of the spin-crossover solid Co(H2(fsa)2en)(py)2 to a pulsed magnetic field. Physical Review B - Condensed Matter and Materials Physics. 2002;65(17):1724121-1724124.

[2] Cavallini M, Bergenti I, Milita S, Ruani G, Salitros I, Qu ZR, et al. Micro- and nanopatterning of spin-transition compounds into logical structures. Angewandte Chemie International Edition. 2008;47(45):8596-8600.

[3] Kahn O, Kröber J, Jay C. Spin transition molecular materials for displays and data recording. Advanced Materials. 1992;4(11):718-728.

[4] Kahn O, Martinez CJ. Spin-transition polymers: From molecular materials toward memory devices. Science. 1998;279(5347):44-48.

[5] Ruben M, Rojo J, Romero-Salguero FJ, Uppadine LH, Lehn JM. Grid-type metal ion architectures: Functional metallosupramolecular arrays. Angewandte Chemie - International Edition. 2004;43(28):3644-3662.

[6] Rubio M, Hernández R, Nogales A, Roig A, López D. Structure of a spin-crossover Fe(II)1,2,4-triazole polymer complex dispersed in an isotactic polystyrene matrix. European Polymer Journal. 2011;47(1):52-60.

[7] Rubio M, López D. Effect of solvent on the gelation properties of a metallo-organic polymer of $[\mathrm{Fe}(\mathrm{II}) \quad$ (4-octadecyl-1,2,4-triazole)3(ClO4)2]n. European Polymer Journal. 2009;45(12):3339-3346.

[8] Roubeau O. Triazole-Based One-Dimensional Spin-Crossover Coordination Polymers. Chemistry-a European Journal. 2012;18(48):15230-15244.

[9] Gural'skiy IyA, Quintero CM, Molnar G, Fritsky IO, Salmon L, Bousseksou A. Synthesis of Spin-Crossover Nano- and Micro-objects in Homogeneous Media. Chemistry-a European Journal. 2012;18(32):9946-9954.

[10] Beattie JK. Dynamics of Spin Equilibria in Metal Complexes. Advances in Inorganic Chemistry. 1988;32(C):1-53. 
[11] Fujigaya T, Jiang DL, Aida T. Switching of Spin States Triggered by a Phase Transition: Spin-Crossover Properties of Self-Assembled Iron(II) Complexes with Alkyl-Tethered Triazole Ligands. Journal of the American Chemical Society. 2003;125(48):14690-14691.

[12] Fujigaya T, Jiang DL, Aida T. Spin-crossover dendrimers: Generation number-dependent cooperativity for thermal spin transition. Journal of the American Chemical Society. 2005;127(15):5484-5489.

[13] Spiering H, Kohlhaas T, Romstedt H, Hauser A, Bruns-Yilmaz C, Kusz J, et al. Correlations of the distribution of spin states in spin crossover compounds. Coordination Chemistry Reviews. 1999;190-192:629-647.

[14] Craig GA, Sanchez Costa J, Roubeau O, Teat SJ, Aromi G. Local Coordination Geometry and Spin State in Novel FeII Complexes with 2,6-Bis(pyrazol-3-yl)pyridine-Type Ligands as Controlled by Packing Forces: Structural Correlations. Chemistry-a European Journal. 2012;18(37):11703-11715.

[15] Armand F, Badoux C, Bonville P, Ruaudel-Teixier A, Kahn O. Langmuir-Blodgett films of spin transition iron(II) metalloorganic polymers. 1. Iron(II) complexes of octadecyl-1,2,4triazole. Langmuir. 1995;11(9):3467-3472.

[16] Soyer H, Mingotaud C, Boillot ML, Delhaes P. Spin crossover of a Langmuir-Blodgett film based on an amphiphilic iron(II) complex. Langmuir. 1998;14(20):5890-5894.

[17] Cobo S, Molnár G, Real JA, Bousseksou A. Multilayer sequential assembly of thin films that display room-temperature spin crossover with hysteresis. Angewandte Chemie International Edition. 2006;45(35):5786-5789.

[18] Bousseksou A, Molnár G, Demont P, Menegotto J. Observation of a thermal hysteresis loop in the dielectric constant of spin crossover complexes: Towards molecular memory devices. Journal of Materials Chemistry. 2003;13(9):2069-2071.

[19] Coronado E, Galán-Mascarós JR, Monrabal-Capilla M, García-Martínez J, Pardo-Ibáñez P. Bistable spin-crossover nanoparticles showing magnetic thermal hysteresis near room temperature. Advanced Materials. 2007;19(10):1359-1361.

[20] Fujigaya T, Jiang DL, Aida T. Spin-crossover physical gels: A quick thermoreversible response assisted by dynamic self-organization. Chemistry - An Asian Journal. 2007;2(1):106113.

[21] Jones JL, Marques CM. Rigid polymer network models. Journal de physique Paris. 1990;51(11):1113-1127.

[22] Lee CS, Kimizuka N. Solvatochromic nanowires self-assembled from cationic, chlorobridged linear platinum complexes and anionic amphiphiles. Chemistry Letters. 2002(12):12521253.

[23] Matsukizono H, Kuroiwa K, Kimizuka N. Self-assembly-directed spin conversion of iron(II) 1,2,4-triazole complexes in solution and their effect on photorelaxation processes of fluorescent counter ions. Chemistry Letters. 2008;37(4):446-447.

[24] Seiffert S, Sprakel J. Physical chemistry of supramolecular polymer networks. Chemical Society Reviews. 2012;41(2):909-930.

[25] Roubeau O, Colin A, Schmitt V, Clérac R. Thermoreversible gels as magneto-optical switches. Angewandte Chemie - International Edition. 2004;43(25):3283-3286.

[26] Grondin P, Roubeau O, Castro M, Saadaoui H, Colin A, Clérac R. Multifunctional gels from polymeric spin-crossover metallo-gelators. Langmuir. 2010;26(7):5184-5195.

[27] Heenan RK, Penfold J, King SM. SANS at Pulsed Neutron Sources: Present and Future Prospects. Journal of Applied Crystallography. 1997;30(6):1140-1147.

[28] Mohan SD, Mitchell GR, Davis FJ. Chain extension in electrospun polystyrene fibres: A SANS study. Soft Matter. 2011;7(9):4397-4404.

[29] Heenan RK, King SM, Osborn R, Stanley HB. COLETTE Users Guide, Rutherford Appleton Laboratory Report RAL-89-128. 1989.

[30] King SM, Heenan RK. Using COLETTE, Rutherford Appleton Laboratory Report RAL95-005. 1995.

[31] Heenam RK. FISH Reference Manual, RAL REPORT 89-129. 1989.

[32] Lin MY, Hanley HJM, Muzny CD, Straty GC. Simultaneous measurement of viscosity and structure for rod-like micelle solutions. Physica B: Condensed Matter. 1997;241-243:990-992. 
[33] Glatter O, Kratky O. Small Angle X-ray Scattering. 1982.

[34] Guenet JM. J Phys II. 1994;4:1077-1082.

[35] Dasgupta D, Kamar Z, Rochas C, Dahmani M, Mesini P, Guenet JM. Design of hybrid networks by sheathing polymer fibrils with self-assembled nanotubules. Soft Matter. 2010;6(15):3573-3581.

[36] Fournet G. Bull Soc Fr Mineral Cristallogr. 1951;74.

[37] Pringle OA, Schmidt PW. J Appl Crystallogr. 1971;4:290-293.

[38] Kuroiwa K, Shibata T, Takada A, Nemoto N, Kimizuka N. Heat-Set Gel-like Networks of Lipophilic Co(II) Triazole Complexes in Organic Media and Their Thermochromic Structural Transitions. Journal of the American Chemical Society. 2004;126(7):2016-2021.

[39] Cates ME. Reptation of living polymers: Dynamics of entangled polymers in the presence of reversible chain-scission reactions. Macromolecules. 1987;20(9):2289-2296.

[40] Cates ME. Dynamics of living polymers and flexible surfactant micelles: Scaling laws for dilution. J Phys (Paris). 1988;49:1593-1600.

[41] Lopez D, Guenet JM. Encapsulation of filaments of a self-assembling bicopper complex in polymer nanowires. European Physical Journal B. 1999;12(3):405-411.

[42] López D, Guenet JM. Behavior of a self-assembling bicopper complex in organic solutions. Macromolecules. 2001;34(4):1076-1081.

[43] Oster G, Riley DP. Scattering from cylindrically symmetric systems. Acta Crystallogr. $1952 ; 5$.

[44] Guenet JM. Thermoreversible Gelation of Polymers and Biopolymers. 1992. 
Structure of a spin-crossover Fe(II)-1,2,4-triazole polymer complex gel in toluene. Small angle neutron scattering and viscoelastic studies

Coro Echeverría, Miguel Rubio, Geoffrey R. Mitchell and Daniel López

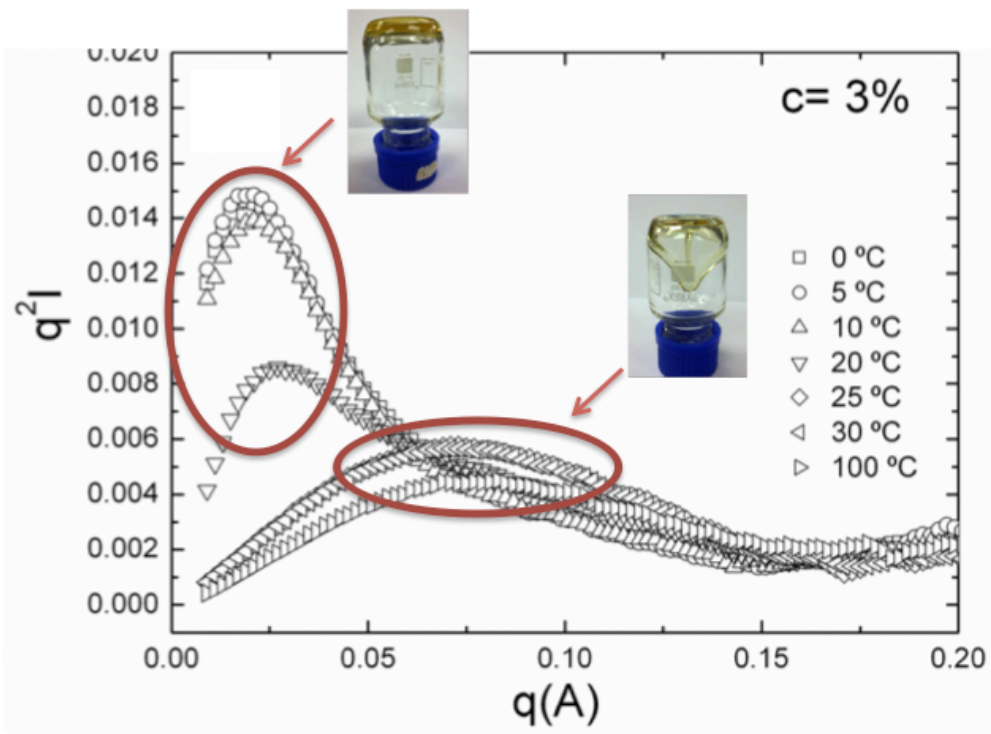

